\title{
Hemorrhagic Encephalopathy in Dengue Shock Syndrome: A Case Report
}

\author{
Luiz José de Souza ${ }^{1,2}$, André Luiz de \\ Oliveira Martins ${ }^{3}$, Paulo César Leitão \\ Paravidini, $^{1,2}$, Rita Maria Ribeiro Nogueira ${ }^{4}$, \\ Carlos Gicovate Neto ${ }^{1,2}$, Diogo Assed Bastos ${ }^{1,2}$, \\ Edno Wallace da Silva Siqueira ${ }^{1,2}$ and \\ Rodrigo da Costa Carneiro ${ }^{1,2}$
}

\begin{abstract}
Dengue Reference Center ${ }^{l}$, The Brazilian Society of Internal Medicine $^{2}$; Medical Intensive Care Unit, Pro Clinicas Hospital ${ }^{3}$; Campos dos Goytacazes, RJ; Department of Virology, Oswaldo Cruz Institute, FIOCRUZ; , Rio de Janeiro, RJ, Brazil.
\end{abstract}

\begin{abstract}
Dengue fever is the most important arboviral infection in the world, with an estimated 100 million cases per year and 2.5 billion people at risk. Encephalopathy is a rare complication of dengue virus infection and may occur as a consequence of intracranial hemorrhage, cerebral edema, hyponatremia, cerebral anoxia, fulminant hepatic failure with portosystemic encephalopathy, microcapillary hemorrhage or release of toxic products. We report a rare case of hemorrhagic encephalopathy in dengue shock syndrome caused by type 3 dengue virus.

Key Words: Dengue fever, encephalopathy, cerebral hemorrhage.
\end{abstract}

Dengue fever is the most important arboviral infection in the world, with an estimated 100 million cases per year and 2.5 billion people at risk [1]. This disease has become an important issue in public health in Brazil since 1986, when serotype 1 of the virus (DENV-1) was detected in the state of Rio de Janeiro [2].

From January to May 2002, Rio de Janeiro state was affected by an epidemic of dengue fever that resulted in 255,483 notified cases and 91 deaths. The predominant strain was viral type 3 (DENV-3) [3]. Approximately one year later, the epidemic that was principally affecting the city of Rio de Janeiro spread to other areas, and DENV-3 was detected in the northern region of the state. During this period, 1,585 cases of the disease were confirmed by the Dengue Fever Reference Center (CRD) in Campos dos

Received on 03 February 2005; revised 02 June 2005.

Address for correspondence: Dr. Luis José de Souza. Sociedade Brasileira de Clínica Médica / RJ. Avenida Alberto Torres, 217 - Centro. Zip code: 28035-580. Campos dos Goytacazes - Rio de Janeiro - Brazil. Telephone/fax number: (22) 27239243 . Email-sbcm.rol@terra.com.br

The Brazilian Journal of Infectious Diseases 2005;9(3):257-261 (C) 2005 by The Brazilian Journal of Infectious Diseases and Contexto Publishing. All rights reserved.
Goytacazes (RJ) [4] and one case of dengue shock syndrome (DSS) involving hemorrhagic encephalopathy was registered.

Various atypical manifestations of dengue virus infection, including involvement of the central nervous system, cardiac disturbances and elevated levels of aminotransferases, with reactive hepatitis, have been reported during recent years [1,5-7]. Encephalopathy is a rare complication of the dengue virus infection and may occur due to intracranial hemorrhage, cerebral edema, hyponatremia, cerebral anoxia, fulminant hepatic failure with portosystemic encephalopathy, microcapillary hemorrhage or release of toxic products.

We examined a case of hemorrhagic encephalopathy in a female patient with DSS caused by DENV-3.

\section{Case Report}

The patient was a 21-year old female student from Campos dos Goytacazes. Her condition began with a high fever $\left(39^{\circ} \mathrm{C}\right)$, cephalea, retroorbital pain, myalgia, arthralgia, watery diarrhea and nausea. Twenty-four hours later, she sought medical treatment and was submitted to a complete blood cell count and chest Xray. No abnormality was detected in these examinations 
and the patient was prescribed symptomatic medication (paracetamol). On the fourth day of her illness, the patient's clinical condition had deteriorated and she returned to the hospital, presenting fever, myalgia, epistaxis, hematemesis, centrifugal pruriginous morbiliforme exanthema and difficulty in moving her lower limbs.

The patient underwent a physical examination and was found to be oriented, with normal skin color (no paleness, no icteric coloration, acyanotic), dyspneic and dehydrated +/4+. Cardiovascular System: regular rhythm, normal first and second heart sounds, blood pressure 100x70 mmHg, heart rate $160 \mathrm{bpm}$. Respiratory System: a generally audible vesicular sound with no adventitious sounds. Her abdomen was flaccid and painful to palpation at the right hypochondrium, and there were no visceromegalies. There was no swelling in her lower limbs. Neurological examination revealed no changes in cranial pairs, preserved superficial and deep sensitivity, flaccid paraparesis and plantar skin reflex in bilateral flexion.

On the sixth day of illness, the patient's clinical condition deteriorated further and she presented psychomotor agitation, arterial hypotension and acute respiratory failure. The patient was referred to the intensive care unit, where she was sedated, intubated and connected to a breathing machine. She presented hypoxia, with a $\mathrm{PaO}_{2} / \mathrm{FiO}_{2}$ ratio of 71 . Chest X-ray revealed bilateral alveolar infiltration (Figure 1) compatible with Adult Respiratory Distress Syndrome (ARDS). She also presented refractory shock to volume infusion, acute hepatitis, acute kidney failure requiring an amine vasopressor (noradrenalin) and hemodialysis. The results of laboratory tests are shown in Table 1.

On the $12^{\text {th }}$ day of the illness, sedation was suspended and 24 hours later the patient spontaneously opened her eyes. She presented right peripheral facial paralysis and flaccid tetraparesis. Computerized tomography of the cranium and magnetic nuclear resonance were carried out, revealing a pontine hematoma (Figures 2-4). There was an improvement in respiratory, cardiovascular, renal and liver function and the patient was discharged from the hospital 30 days after admission. After six months of out-patient follow-up, the patient was lucid and oriented, but was walking with difficulty.

The diagnosis of dengue fever was confirmed by Reverse Transcriptase Polymerase Chain Reaction (RT-PCR); serotype 3 viral strain was identified in the sample collected on the fifth day of the disease. Diagnosis was also confirmed by the presence of IgM antibodies (MACELISA) [2,8]. Serum testing for hepatitis A (anti-HAV IgM), hepatitis B (HBsAg and anti-HBc) and PCR for hepatitis $\mathrm{C}$ were all negative. Serum agglutination for leptospirosis was also negative.

\section{Discussion}

The relationship between hemorrhagic dengue fever (HDF) and neurological disturbances was first described in 1976, and since then several publications have added to the information available on this disease $[1,9]$. Encephalopathy in HDF is an atypical manifestation and may appear in various forms, including depressed sensitivity, convulsion, behavioral disorders, meningeal involvement and focal signs of the virus in the central nervous system $[5,6,10]$.

Physiopathology of neurological involvement may include the following factors: direct tissue lesion caused by the virus, capillary hemorrhage, disseminated intravascular coagulation, metabolic disorder (hyponatremia and metabolic acidosis), fulminant hepatic failure and cerebral edema caused by the increase in vascular permeability [5-7].

In a series of six cases of encephalopathy, Lum et al. (1996) identified the dengue virus in the cerebrospinal fluid of five patients using isolation techniques or PCR and hybridization in situ. The DENV-3 virus strain was isolated in four cases and serotype 2 (DENV-2) in one case, suggesting the neurovirulent property of dengue virus, particularly DENV-3, as one of the etiopathogenic factors responsible for affecting the nervous system [6,7].

The involvement of the central nervous system by DENV-1 was demonstrated during the epidemic that 
Table 1. Laboratory data of a dengue shock syndrome patient

\begin{tabular}{lccc}
\hline Laboratory Tests & $\mathbf{4}^{\text {th }}$ Day of illness & $\mathbf{6}^{\text {th }}$ day of illness & $\mathbf{1 2}^{\text {th }}$ day of illness \\
\hline Leukocytes ( /i工) & 4,210 & 7,400 & 23,800 \\
Ht (\%)/ Hb (g/dL) & $39.7 / 13.6$ & $48.8 / 16.3$ & $35.5 / 12.2$ \\
Platelets (/i工) & 108,000 & 34,000 & 95,000 \\
Urea (mg/dL) & 21 & 38 & 113 \\
Creatinine(mg/dL) & 0.7 & 1.4 & 4.1 \\
AST / ALT (U/L) & - & $1,448 / 587$ & $704 / 379$ \\
PT (seconds)/ INR & $13 / 1.0$ & $15 / 1.24$ & $14 / 1.1$ \\
Albumin(g/dL) & - & 2.2 & 2.6 \\
Lactate (mg/dL) & - & 96 & 18.1 \\
Glycemia (mg/dL) & 72 & 78 & 126 \\
Sodium (mEq/L) & 140 & 136 & 128 \\
Potassium(mEq/L) & 3.8 & 3.3 & 4.7 \\
\hline
\end{tabular}

Ht: hematocrit; Hb: hemoglobin; AST: aspartate aminotransferase; ALT: alanine aminotransferase; PT: prothrombin time; INR: International Normalized Ratio.

Figure 1. Adult Respiratory Distress Syndrome (ARDS). 
Figure 2. Computerized Cranial Tomography (CCT) - pontine hematoma.

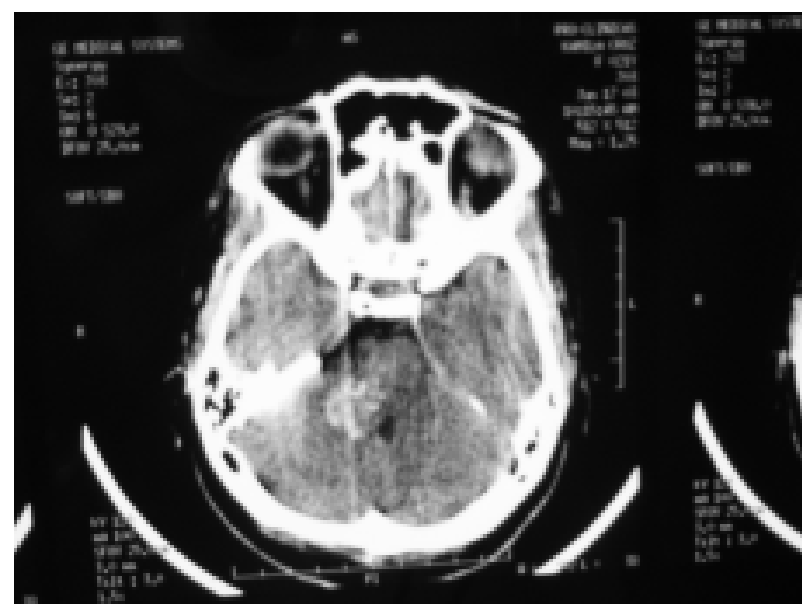

Figure 3. Nuclear magnetic resonance (NMR) - pontine hemorrhage.

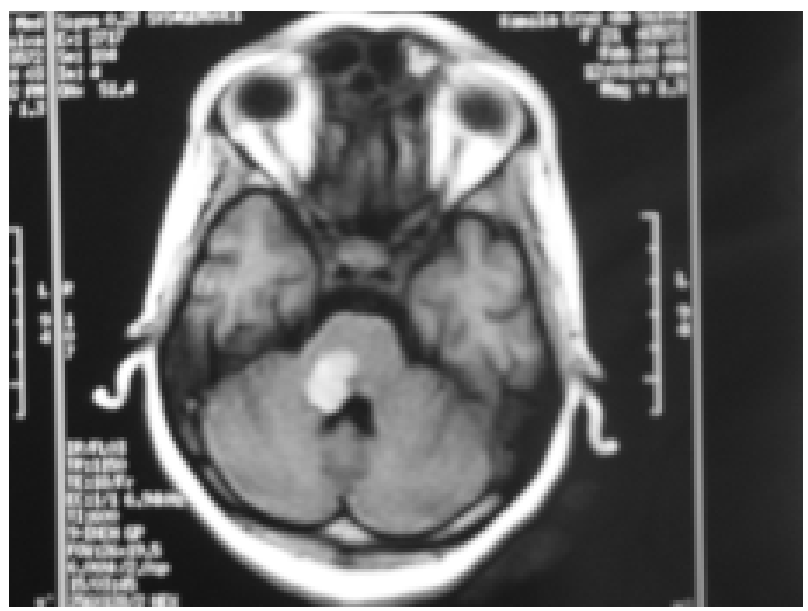

Figure 4. Nuclear magnetic resonance (NMR) - pontine hemorrhage.

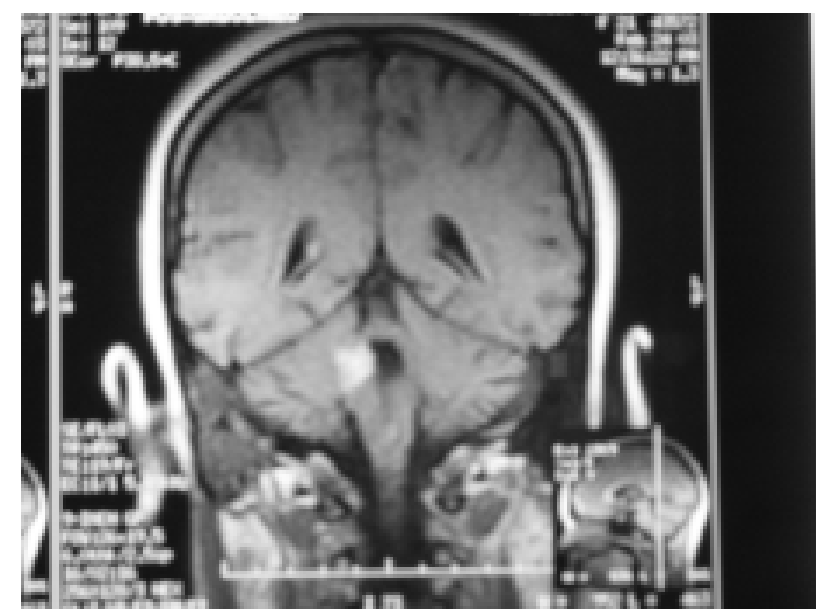

www.bjid.com.br 
occurred in 1986-7 in the state of Rio de Janeiro [11]. Later, DENV-2 was identified in the brain when the specific antigen was detected in the cortical grey matter by immunohistochemistry [12].

In this case report, the patient presented clinical and laboratory findings compatible with $\mathrm{HDF}$, evolving to shock that was refractory to volume infusion, kidney failure, ARDS and acute hepatitis, requiring intensive care. The neurological manifestations were peripheral facial paralysis and flaccid paraparesis evolving to tetraparesis. Viral isolation identified viral strain serotype 3 , which has been associated with neurological manifestations. Cranial tomography and magnetic nuclear resonance showed evidence of pontine hemorrhage (Figures 2 and 3).

Despite the severity of this patient's clinical condition, she recovered her organic functions and is currently undergoing physiotherapy to recover motor function.

\section{Conclusion}

Encephalopathy is a rare manifestation of HDF. It should, however, be investigated in endemic areas and during epidemics of dengue, since early diagnosis is essential for its management. Hemorrhagic complications and circulatory collapse are determinant factors in the prognosis and are the principal causes of death in these patients.

\section{References}

1. Solomon T., Dung N.M., Vaughn D.W., et al. Neurological Manifestations of dengue infection. The Lancet Mar 2000;355:1053-9.

2. Lanciotti R.S., Calisher C.H., Gubler D.J., et al. Rapid detection and typing of dengue viruses from clinical samples by using reverse transcriptase-polymerase chain reaction. J Clin Microbiol 1992;30:545-51.

3. Secretaria de Estado de Saúde do Rio de Janeiro (SES/ RJ). Quadro demonstrativo de casos notificados de dengue no estado do Rio de Janeiro de 1986-2002. Rio de Janeiro, Brasil; 2002.
4. Souza L.J., Alves G.J., Nogueira S.A., et al. Aminotransferase and Acute Hepatitis in Patients with Dengue Fever: Analysis of 1,585 Cases. BJID 2004;8:156-63.

5. Kho L.K., Sumarmo H.; Wulur E.C., et al. Dengue Hemorrhagic Fever Accompanied by Encephalopathy in Jakarta. Southeast Asian J Trop Med Public Health 1981; $12: 83-6$.

6. Lum L.C., Lam S.K., Choy Y.S., et al. Dengue Encephalitis: a True Entity? Am J Trop Med Hyg 1996;54:256-59.

7. Angibaud G., Luaute J., Laille M., et al. Brain Involvement in Dengue Fever. Journal of Clinical Neuroscience 2001;8:63-5.

8. Kuno G., Gomez I., Gubler D.J. Detecting artificial antidengue IgM immune complexes using an enzyme linked immunosorbent assay. Am J Trop Med Hyg 1987;36:153-9.

9. Pancharoen C., Thisyakorn U. Neurological Manifestations in Dengue Patients. Southeast Asian J Trop Med Public Health 2001;32:341-45..

10. Thakare J., Walhecar B., Banerjee K. Hemorrhagic Manifestations and Encephalopathy in Cases of Dengue in India. Southeast Asian J Trop Med Public Health 1996;27:471-75.

11. Miagostovich M.P., Ramos R.G., Nicol A.F., et al. Retrospective study on dengue fatal cases. Clinical Neuropath 1997; 16:204-8.

12. Nogueira R.M.R., Filippis A.M.B., Coelho J.M.O., et al. Dengue virus infection of the central nervous systems (CNS): a case report from Brazil. South. Asian J Trop Med Public Health 2002;33:68-71. 